\title{
Hybrid Synchronization of General T-S Fuzzy Complex Dynamical Networks with Time-Varying Delay
}

\author{
Degang Xu, Weihua Gui, Panlei Zhao, and Chunhua Yang \\ College of Information Science and Engineering, Central South University, Changsha 410083, China \\ Correspondence should be addressed to Degang Xu; dgxu@csu.edu.cn
}

Received 5 April 2013; Accepted 28 April 2013

Academic Editor: Wenwu Yu

Copyright (c) 2013 Degang Xu et al. This is an open access article distributed under the Creative Commons Attribution License, which permits unrestricted use, distribution, and reproduction in any medium, provided the original work is properly cited.

\begin{abstract}
We propose and investigate a new general model of fuzzy complex network systems described by Takagi-Sugeno (T-S) fuzzy model with time-varying delays. Hybrid synchronization problem is discussed for this general T-S fuzzy complex dynamical network with nondelayed and delayed coupling between nodes. Utilizing Lyapunov-Krasovskii functional method, synchronization stability criteria for the networks are established in terms of linear matrix inequalities (LMIs). These criteria reveal the relationship between coupling matrices with time-varying delays and synchronization stability of the dynamical network. Numerical simulation is provided to illustrate the effectiveness and advantage of derived theoretical results.
\end{abstract}

\section{Introduction}

In recent years, complex dynamical networked agent systems have attracted a great deal of attention in various engineering fields from physics to biology, chemistry, and computer science [1-3]. The reason can be attributed to their flexibility and generality for representing virtually any natural and manmade systems. Such systems in the real world usually consist of a large number of highly interconnected dynamical units. Transportation networks, coupled biological and chemical engineering systems, neural networks in human brains, and the Internet are only a few of such examples [4].

Synchronization is one of the most significant and interesting collective behaviors in complex networked agent systems due to its potential applications in many fields including secure communization, parallel image processing, and information science [5-7]. On the other hand, time delays occur commonly in complex networks because of the finite speed of signal transmission over the links [8]. Since the time delay often causes undesirable performance and instability of the network, various approaches to synchronization analysis for complex dynamical networks with time delay have been investigated in the literature [9-12]. Therefore, synchronization criteria of complex networks with delays have become a topic of practical importance. The stability criteria for time delay systems include two categories: delay-dependent ones and delay-independent ones. Since delay-dependent stability criteria include the information on the size of delay, delay-dependent stability criteria are generally less conservative than delay-independent ones [10]. Moreover, many real-world networks are not static but more likely to be time-varying evolving, particularly in biological and physical networks. Commonly, time-varying delays are general form of time delays. There are a few research works [13-15] considering the time-varying coupling for complex agent systems of dynamical networks.

Furthermore, the uncertainty or vagueness is unavoidable in real modeling problems of agent systems. Fuzzy theory as an efficient tool in approximating a complex nonlinear system is a feasible method to take vagueness into consideration [16]. There are some research works investigating the problem of delay-dependent robust controllers and filtering design for a class of uncertain state-delayed Takagi-Sugeno (T-S) fuzzy systems [17-20]. Recently, the problems of stability analysis, approximation, and stabilization for Takagi-Sugeno fuzzy systems with time-varying state delay are investigated [21-24]. So, fuzzy complex networks have advantages over pure complex networks since they incorporate the capability of fuzzy reasoning in handling uncertain information. In this regard, the fuzzy models to describe complex dynamical 
networks which are subjected to nonlinearity and have timevarying delays are introduced. The synchronization problem for T-S fuzzy stochastic discrete-time complex networks with mixed time-varying delays is discussed in a recent work [25]. By employing the information of probability distribution of time delays, the original system is transformed into a T-S fuzzy model with stochastic parameter [26, 27]. But how to solve the synchronization problem for general fuzzy complex networks still remains largely unsolved and challenging. To the best of our knowledge, time-varying delay [28, 29] synchronization analysis of general fuzzy complex dynamical networks has not been reported in the literature.

Besides, it is noticed that most of the studies on synchronization of dynamical networks have been performed under some implicit assumptions that there exists the information communication of nodes via the edges only at time $t$ or at time $t_{-} \tau$ [30]. However, in many circumstances, this simplification does not match satisfactorily the peculiarities of real networks; there exists the information communication of nodes not only at time $t$ but also at time $t_{-} \tau$. More recently, coexistence of the hybrid synchronization in chaotic systems was investigated intensively [31]. However, our generalized dynamical network model has different coupling strengths for different connections. So it is complex and extended comparing with the simplified network model in [32]. So our conclusion is more compact and more meaningful for the generalized network models. To the best of our knowledge, there are very few studies on the hybrid synchronization of general coupled complex dynamical networks with nondelayed and delayed coupling in the literature.

Motivated by the previous discussions, in this paper, we attempt to introduce some more general time-varying dynamical network models based on the T-S fuzzy model and investigate the synchronization properties of this model. Based on the Lyapunov-Krasovskii functional method, we use a linearized method to solve the problem of synchronization for fuzzy complex networks with time-varying coupling delay and derive hybrid synchronization conditions for delaydependent stabilities in terms of LMIs, the time-varying network model. A numerical example is given to demonstrate the effectiveness and the advantage of the proposed method.

The rest of this paper is organized as follows. In Section 2, we present some preliminaries for proving the proof and the fuzzy time-varying coupled dynamical network model. Hybrid synchronization criterions are derived in Section 3. In Section 4, we provide a numerical simulation to verify the correctness and effectiveness of the derived results. Conclusions are presented in Section 5.

\section{Problem Formulation}

Consider the following model of general continuous-time complex networks with time-varying coupling delays which can be represented by a T-S fuzzy model.
Rule $l:$ If $\theta_{1}(t)$ is $F_{l 1}, \theta_{2}(t)$ is $F_{l 2}, \ldots, \theta_{g}(t)$ is $F_{l g}$, then

$$
\begin{gathered}
\dot{x}_{i}(t)=f\left(x_{i}(t)\right)+\varepsilon \sum_{j=1}^{N} c_{i j} \Gamma_{l} x_{j}(t) \\
+\varepsilon \sum_{j=1}^{N} \widetilde{c}_{i j} \widetilde{\Gamma}_{l} x_{j}(t-\tau(t)), \\
t>0, \quad i=1,2, \ldots, N, \\
x(t)=\phi(t), \quad t \in[-h, 0],
\end{gathered}
$$

where $F_{l j}(l=1, \ldots, r ; j=1, \ldots, g)$ are the fuzzy sets; $r$ is the number of rules and $\theta_{j}(j=1, \ldots, g)$ are the premise variables; $N$ is the number of agent nodes, where each agent node is an $n$-dimensional dynamical system with node dynamics $\dot{x}=f(x, t) ; x_{i}=\left(x_{i 1}, x_{i 2}, \ldots, x_{i n}\right)^{T} \epsilon$ $R^{n}$ are the state variables of node $i ; f: R^{n} \rightarrow R^{n}$ is continuously differentiable; $\Gamma_{l}$ and $\widetilde{\Gamma}_{l}$ are the constant innercoupling matrices of the nodes; the constant $\varepsilon>0$ is the coupling strength; $C=\left(c_{i j}\right)_{N \times N}$ and $\widetilde{C}=\left(\widetilde{c}_{i j}\right)_{N \times N}$ are the outer-coupling matrices of the network, in which $c_{i j}$ is defined, as follows: if there is a connection between node $i$ and node $j(j \neq i)$, then $c_{i j}=c_{j i}=1$; otherwise, $c_{i j}=c_{j i}=0$, $\widetilde{c}_{i j}$ is similar defined and the diagonal elements of matrices $C$ and $\widetilde{C}$ are defined by

$$
\begin{gathered}
c_{i j}=-\sum_{j=1, i \neq j}^{N} c_{i j}=-\sum_{j=1, i \neq j}^{N} c_{j i}, \\
\widetilde{c}_{i j}=-\sum_{j=1, i \neq j}^{N} \widetilde{c}_{i j} \\
=-\sum_{j=1, i \neq j}^{N} \widetilde{c}_{j i}, \quad i=1, \ldots, N .
\end{gathered}
$$

Suppose that $\mathrm{C}$ and $\widetilde{C}$ are irreducible matrices. Time-varying delay $\tau(t)$ satisfies

$$
0 \leq \tau(t) \leq h, \quad \dot{\tau}(t) \leq \gamma
$$

in which $h$ and $\gamma$ are constants. The initial function $\phi(t)$ is a continuous and differentiable vector-valued function.

By using the standard fuzzy inference method, the T-S fuzzy network (1) can be expressed by the following model:

$$
\begin{array}{r}
\dot{x}_{i}(t)=\sum_{l=1}^{r} \mu_{l}(\theta(t))\left[f\left(x_{i}(t)\right)+\varepsilon \sum_{j=1}^{N} c_{i j} \Gamma_{l} x_{j}(t)\right. \\
\left.+\varepsilon \sum_{j=1}^{N} \widetilde{c}_{i j} \widetilde{\Gamma}_{l} x_{j}(t-\tau(t))\right],
\end{array}
$$

where $\theta(t)=\left[\theta_{1}(t), \theta_{2}(t), \ldots, \theta_{g}(t)\right]$, and $\mu_{l}(\theta(t))=\omega_{l}(\theta(t)) /$ $\sum_{l=1}^{r} \omega_{l}(\theta(t))$, in which $F_{l j}\left(\theta_{j}(t)\right)$ is the grade of membership 
of $\theta_{j}(t)$ in $F_{l j}$. It is obvious that the fuzzy weighting functions $\mu_{l}(\theta(t))$ satisfy

$$
\mu_{l}(\theta(t)) \geq 0, \quad \sum_{l=1}^{r} \mu_{l}(\theta(t))=1 .
$$

In the following, some elementary situations are introduced, which play an important role in proving the main result.

Definition 1. The dynamical networked agent system (1) is said to achieve asymptotic synchronization if

$$
x_{1}(t)=x_{2}(t)=\cdots=x_{N}(t)=s(t), \quad t \rightarrow \infty,
$$

where $s(t)$ is a solution of an isolate node, satisfying $\dot{s}(t)=$ $f(s(t))$.

Lemma 2 (see [7]). If $C=\left(c_{i j}\right)_{N \times N}$ satisfies the aforementioned defined conditions, then there exists a unitary matrix, $\phi=\left(\phi_{1}, \ldots, \phi_{N}\right)$, such that $C^{T} \phi_{k}=\lambda_{k} \phi_{k}, k=1,2, \ldots, N$, where $\lambda_{k}, k=1,2, \ldots, N$, are the eigenvalues of matrix $C$.

Lemma 3 (see [12]). Let $X$ and $Y$ be arbitrary $n$-dimensional real vectors, and let $K$ be an $n \times n$ positive definite matrix. $P \in R^{n \times n}$ is an arbitrary real matrix. Then, the following matrix inequality holds:

$$
2 X^{T} P Y \leq X^{T} P K^{-1} P^{T} X+Y^{T} K Y .
$$

The aim of this paper is to investigate synchronization problem of the fuzzy complex dynamical network with timevarying delay (4).

\section{Main Results}

In this section, we focus on investigating the hybrid synchronization problem of fuzzy complex dynamical networks with nondelayed and delayed coupling. Before deriving our main results, the following lemma will be utilized.

Lemma 4. Consider the T-S fuzzy dynamical network (4). Let $0=\lambda_{1}>\lambda_{2} \geq \cdots \geq \lambda_{N}$ and $0=\tilde{\lambda}_{1}>\tilde{\lambda}_{2} \geq \cdots \geq \tilde{\lambda}_{N}$, respectively, be the eigenvalues of outer coupling matrix $C$ and $\widetilde{C}$. If the following $N-1$ time-varying delayed differential equations are asymptotically stable about their zero solution:

$$
\begin{array}{r}
\dot{w}_{k}(t)=\sum_{l=1}^{r} \mu_{l}(\theta(t))\left[\left(J(t)+\varepsilon \lambda_{k} \Gamma_{l}\right) w_{k}(t)\right. \\
\left.+\varepsilon \widetilde{\lambda}_{k} \widetilde{\Gamma}_{l} w_{k}(t-\tau(t))\right], \\
k=2,3, \ldots, N,
\end{array}
$$

where $J(t)$ is the Jacobin of $f(x(t))$ at $s(t)$, then synchronized states in fuzzy complex networks (1) are asymptotically stable.

Proof. For the synchronized states of complex networks (4), we have

$$
x_{i}(t)=s(t)+e_{i}(t), \quad i=1,2, \ldots, N \text {. }
$$

Substituting (9) into (4), we obtain

$$
\begin{array}{r}
\dot{e}_{i}(t)=\sum_{l=1}^{r} \mu_{l}(\theta(t))\left[f\left(s(t)+e_{i}(t)\right)-f(s(t))\right. \\
+\varepsilon \sum_{j=1}^{N} c_{i j} \Gamma_{l} e_{j}(t) \\
\left.+\varepsilon \sum_{j=1}^{N} \widetilde{c}_{i j} \widetilde{\Gamma}_{l} e_{j}(t-\tau(t))\right] .
\end{array}
$$

Considering that $f(s(t))$ is continuous differentiable, it is easy to know that the origin of the complex networks (8) is an asymptotically stable equilibrium point for the following linear time delay systems:

$$
\begin{gathered}
\dot{e}_{i}(t)=\sum_{l=1}^{r} \mu_{l}(\theta(t))\left[J(t) e_{i}(t)+\varepsilon \sum_{j=1}^{N} c_{i j} \Gamma_{l} e_{j}(t)\right. \\
\left.+\varepsilon \sum_{j=1}^{N} \widetilde{c}_{i j} \widetilde{\Gamma}_{l} e_{j}(t-\tau(t))\right] \\
=\sum_{l=1}^{r} \mu_{l}(\theta(t)) \\
\times\left[J(t) e_{i}(t)+\varepsilon \Gamma_{l}\left(e_{1}(t), \ldots, e_{N}(t)\left(c_{i 1}, \ldots, c_{i N}\right)^{T}\right.\right. \\
+\varepsilon \widetilde{\Gamma}_{l}\left[e_{1}(t-\tau(t)), \ldots, e_{N}(t-\tau(t)]\right. \\
\left.\times\left(\widetilde{c}_{i 1}, \ldots, \widetilde{c}_{i N}\right)^{T}\right] .
\end{gathered}
$$

Let $e(t)=\left(e_{1}(t), \ldots, e_{N}(t)\right) \in R^{N \times N}$; we can obtain

$\dot{e}_{i}(t)$

$$
=\sum_{l=1}^{r} \mu_{l}(\theta(t))\left[J(t) e_{i}(t)+\varepsilon \Gamma_{l} e(t) C^{T}+\varepsilon \widetilde{\Gamma}_{l} e(t-\tau(t)) \widetilde{C}^{T}\right] .
$$

According to Lemma 2, there exist nonsingular matrices, $\Phi_{1}=\left(\phi_{11}, \ldots, \phi_{1 N}\right), \Phi_{2}=\left(\phi_{21}, \ldots, \phi_{2 N}\right)$, such that $C^{T} \Phi_{1}=$ $\Phi_{1} \Lambda_{1}$ and $\widetilde{C}^{T} \Phi_{2}=\Phi_{2} \Lambda_{2}$, with $\Lambda_{1}=\operatorname{diag}\left(\lambda_{1}, \ldots, \lambda_{N}\right), \Lambda_{2}=$ $\operatorname{diag}\left(\widetilde{\lambda}_{1}, \ldots, \tilde{\lambda}_{N}\right)$. Using the nonsingular transform $e(t) \Phi=$ $w(t), e(t-\tau) \Phi=w(t-\tau)$, then we obtain

$$
\begin{array}{r}
\dot{w}_{k}(t)=\sum_{l=1}^{r} \mu_{l}(\theta(t))\left[\left(J(t)+\varepsilon \lambda_{k} \Gamma_{l}\right) w_{k}(t)\right. \\
\left.+\varepsilon \widetilde{\lambda}_{k} \widetilde{\Gamma}_{l} w_{k}(t-\tau(t))\right], \\
k=1, \ldots, N .
\end{array}
$$

Note that $\lambda_{1}=0, \tilde{\lambda}_{1}=0$ correspond to the synchronization of the network states (4), where the state $s(t)$ is an orbital 
stable solution of the isolate node as assumed before in (4). If the following $N-1$ pieces of $n$-dimensional linear multiple time delay differential equations

$$
\begin{array}{r}
\dot{w}_{k}(t)=\sum_{l=1}^{r} \mu_{l}(\theta(t))\left[\left(J(t)+\varepsilon \lambda_{k} \Gamma_{l}\right) w_{k}(t)\right. \\
\left.+\varepsilon \widetilde{\lambda}_{k} \widetilde{\Gamma}_{l} w_{k}(t-\tau(t))\right], \\
k=1, \ldots, N
\end{array}
$$

are asymptotically stable, then $e(t)$ will tend to the origin asymptotically, which implies that synchronized states of complex networks (8) are asymptotically stable.

The proof is thus completed.

Remark 5. We use Lemma 4 to linearize the complicated system to deal with the complex networks by using fuzzy theory. It is an available way to investigate the interconnected dynamical agents of complex networks. Then some new subsystems can be obtained, which are easy to be analyzed.

Now, the main result is stated in the following theorem.

Theorem 6. If there exist positive definite symmetric matrices $P_{k}=P_{k}^{T}>0, Q_{k}=Q_{k}^{T}>0, R_{k}=R_{k}^{T}>0$, and $Z_{k}=Z_{k}^{T}>0$, such that the following LMI holds for all $l=1,2, \ldots, r$ :

$$
\left[\begin{array}{ccccc}
\Theta_{k} & h \Omega_{1 k} & h \Omega_{2 k} & h \Omega_{3 k} & h \Pi_{k}^{T} Z_{k} \\
* & -h Z_{k} & 0 & 0 & 0 \\
* & * & -h Z_{k} & 0 & 0 \\
* & * & * & -h Z_{k} & 0 \\
* & * & * & * & -h Z_{k}
\end{array}\right]<0
$$

where

$$
\begin{aligned}
& \Omega_{1 k}=\left[\begin{array}{c}
N_{1 k}^{1} \\
N_{1 k}^{2} \\
N_{1 k}^{2}
\end{array}\right], \quad \Omega_{2 k}=\left[\begin{array}{c}
N_{2 k}^{1} \\
N_{2 k}^{2} \\
N_{2 k}^{2}
\end{array}\right], \quad \Omega_{3 k}=\left[\begin{array}{c}
N_{3 k}^{1} \\
N_{3 k}^{2} \\
N_{3 k}^{2}
\end{array}\right] \\
& \text { for } k=2,3, \ldots, N \text {, } \\
& \Theta_{k}=\Theta_{k 1}+\Theta_{k 2}+\Theta_{k 2}^{T} \\
& =\left[\begin{array}{ccc}
P_{k}\left(J(t)+\varepsilon \lambda_{k} \Gamma_{l}\right)+\left(J(t)+\varepsilon \lambda_{k} \Gamma_{l}\right)^{T} P_{k} & \varepsilon \widetilde{\lambda}_{k} \widetilde{\Gamma}_{l} P_{k} & 0 \\
* & -(1-\gamma) & 0 \\
* & * & -R_{k}
\end{array}\right], \\
& \Theta_{k 2}=\left[\Omega_{k 1}+\Omega_{k 3}-\Omega_{k 1}+\Omega_{k 2}-\Omega_{k 2}-\Omega_{k 3}\right] \text {, } \\
& \Pi_{k}=\left[\begin{array}{lll}
J(t)+\varepsilon \lambda_{k} \Gamma_{l} & \varepsilon \widetilde{\lambda}_{k} \widetilde{\Gamma}_{l} & 0
\end{array}\right],
\end{aligned}
$$

where * denotes the symmetric terms in a symmetric matrix, then the asymptotic synchronization of complex network system (8) can be achieved.
Proof. Construct a Lyapunov-Krasovskii function as

$$
\begin{aligned}
V_{k}(t)= & w_{k}^{T}(t) P_{k} w_{k}(t)+\int_{t-\tau(t)}^{t} w_{k}^{T}(s) Q_{k} w_{k}(s) d s \\
& +\int_{t-h}^{t} w_{k}^{T}(s) R_{k} w_{k}(s) d s \\
& +\int_{-h}^{0} \int_{t+\theta}^{t} \dot{w}_{k}^{T}(s) Z_{k} \dot{w}_{k}(s) d s d \theta .
\end{aligned}
$$

According to Lemma 4, calculating the time derivative of $V_{k}(t)$ along the trajectories of complex networks (8), we obtain

$$
\begin{aligned}
\dot{V}_{k}(t)= & 2 w_{k}^{T}(t) P_{k} \dot{w}_{k}(t)+w_{k}^{T}(t) Q_{k} w_{k}(t) \\
& -(1-\dot{\tau}(t)) w_{k}^{T}(t-\tau(t)) Q_{k} w_{k}(t-\tau(t)) \\
& +w_{k}^{T}(t) R_{k} w_{k}(t)-w_{k}^{T}(t-h) R_{k} \dot{w}_{k}(t-h) \\
& +h \dot{w}_{k}^{T}(s) Z_{k} \dot{w}_{k}(s)-\int_{t-h}^{t} \dot{w}_{k}^{T}(s) Z_{k} \dot{w}_{k}(s) \\
\leq & 2 w_{k}^{T}(t) P_{k} \dot{w}_{k}(t)+w_{k}^{T}(t) Q_{k} w_{k}(t) \\
& -(1-\gamma) w_{k}^{T}(t-\tau(t)) Q_{k} w_{k}(t-\tau(t)) \\
& +w_{k}^{T}(t) R_{k} w_{k}(t)-w_{k}^{T}(t-h) R_{k} \dot{w}_{k}(t-h) \\
& +h \dot{w}_{k}^{T}(s) Z_{k} \dot{w}_{k}(s)-\int_{t-h}^{t} \dot{w}_{k}^{T}(s) Z_{k} \dot{w}_{k}(s) .
\end{aligned}
$$

Based on the Newton-Leibniz formula, the following equations can be obtained for arbitrary matrices $\Xi_{k}$ with appropriate dimensions:

$$
2 \xi_{k}^{T}(t) \Xi_{k}\left[w_{k}(t)-w_{k}(t-\tau)-\int_{t-\tau}^{t} \dot{w}_{k}(s) d s\right]=0,
$$

where $\varsigma_{k}^{T}(t)=\left(w_{k}^{T}(t), w_{k}^{T}(t-\tau)\right)$ and $\Xi_{k}^{T}=\left(X_{1}^{T}, X_{2}^{T}\right)$.

Therefore, using Lemma 3, we have

$$
\begin{aligned}
& \dot{V}_{k}(t)= 2 w_{k}^{T}(t) P_{k} \dot{w}_{k}(t)+w_{k}^{T}(t)\left(Q_{k}+R_{k}\right) w_{k}(t) \\
&-(1-\gamma) w_{k}^{T}(t-\tau(t)) Q_{k} w_{k}(t-\tau(t)) \\
&-w_{k}^{T}(t-h) R_{k} \dot{w}_{k}(t-h)+h \dot{w}_{k}^{T}(t) Z_{k 1} \dot{w}_{k}(t) \\
&-\int_{t-\tau(t)}^{t} \dot{w}_{k}^{T}(s) Z_{k 1}(s) d s \\
&-\int_{t-h}^{t-\tau(t)} \dot{w}_{k}^{T}(s) Z_{k 1} \dot{w}_{k}(s) d s \\
&-\int_{t-h}^{t} \dot{w}_{k}^{T}(s) Z_{k 1} \dot{w}_{k}(s) d s \\
&+2 \Delta_{k}^{T}(t) \Omega_{1 k}\left[w_{k}(t)-w_{k}(t-\tau(t))\right. \\
&\left.-\int_{t-\tau(t)}^{t} \dot{w}_{k}^{T}(s) d s\right]
\end{aligned}
$$




$$
\begin{aligned}
& +2 \Delta_{k}^{T}(t) \Omega_{2 k}\left[w_{k}(t-\tau(t))-w_{k}(t-h)\right. \\
& \left.-\int_{t-h}^{t-\tau(t)} \dot{w}_{k}^{T}(s) d s\right] \\
& +2 \Delta_{k}^{T}(t) \Omega_{3 k}\left[w_{k}(t)-w_{k}(t-h)\right. \\
& \left.-\int_{t-h}^{t} \dot{w}_{k}^{T}(s) d s\right] \\
& <\sum_{i=1}^{r} \mu_{l}(\theta(t))\left\{\Phi _ { k } ^ { T } ( t ) \left[\Theta_{k}+h \widetilde{\Gamma}_{k}^{T} Z_{k} \widetilde{\Gamma}_{k}\right.\right. \\
& +h \Omega_{1 k} Z_{k}^{-1} \Omega_{1 k}^{T} \\
& +h \Omega_{2 k} Z_{k}^{-1} \Omega_{2 k}^{T} \\
& \left.+h \Omega_{3 k} Z_{k}^{-1} \Omega_{3 k}^{T}\right] \Phi_{k}(t) \\
& -\int_{t-\tau(t)}^{t}\left[\Phi_{k}^{T}(t) \Omega_{1 k}+\dot{w}_{k}^{T}(s) Z_{k}\right] Z_{k}^{-1} \\
& \times\left[\Omega_{1 k}^{T} \Phi_{k}(t)+Z_{k} \dot{w}_{k}(s)\right] d s \\
& -\int_{t-h}^{t-\tau(t)}\left[\Phi_{k}^{T}(t) \Omega_{2 k}+\dot{w}_{k}^{T}(s) Z_{k}\right] Z_{k}^{-1} \\
& \times\left[\Omega_{2 k}^{T} \Phi_{k}(t)+Z_{k} \dot{w}_{k}(s)\right] d s \\
& -\int_{t-h}^{t}\left[\Phi_{k}^{T}(t) \Omega_{3 k}+\dot{w}_{k}^{T}(s) Z_{k}\right] Z_{k}^{-1} \\
& \left.\times\left[\Omega_{3 k}^{T} \Phi_{k}(t)+Z_{k} \dot{w}_{k}(s)\right] d s\right\},
\end{aligned}
$$

where $\Delta_{k}^{T}(t)=\left[w_{k}^{T}(t), w_{k}^{T}(t-\tau), w_{k}^{T}(t-h)\right]^{T}$. Because the last three terms are all less than zero, if

$$
\begin{aligned}
\Theta_{k}+ & h \widetilde{\Gamma}_{k}^{T} Z_{k} \widetilde{\Gamma}_{k}+h \Omega_{1 k} Z_{k}^{-1} \Omega_{1 k}^{T}+h \Omega_{2 k} Z_{k}^{-1} \Omega_{2 k}^{T} \\
& +h \Omega_{3 k} Z_{k}^{-1} \Omega_{3 k}^{T}<0,
\end{aligned}
$$

then $\dot{V}_{k}(t)<-\kappa\|w(t)\|^{2}$ for sufficiently small $\kappa>0$. It is easy to see that system (8) is globally synchronized. By Schur complements, we know that the function (21) is equivalent to the function (15).

The proof is thus completed.

Remark 7. For the delay-dependent synchronization problems of complex networks, the Lyapunov-Krasovskii condition has been attracted owing to the structural advantage. The key point is the introduction of the integral inequality technique, a fundamental trick to derive delay-dependent stability criteria containing the size or the bounds of delays and their derivatives. However, almost results in this field have employed partial information on the relationship among delay-related terms. Being differently from the common construction, we use not only the time-varying-delayed

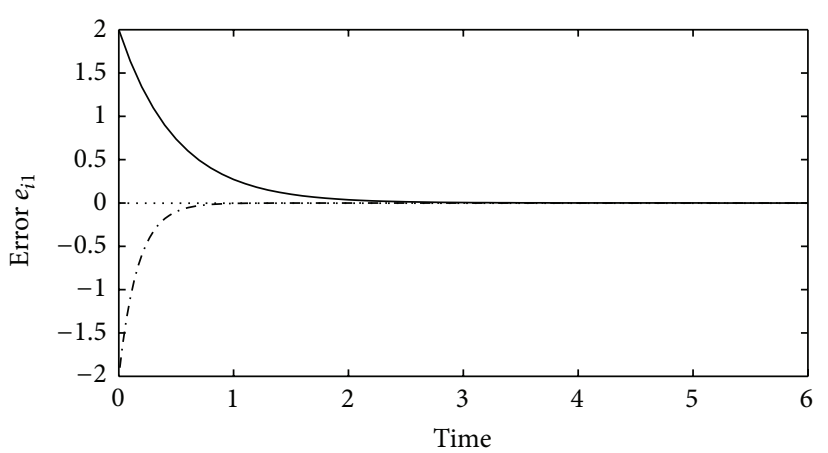

FIGURE 1: Synchronization error $e_{i 1}$ for the coupling delayed networks with $h=\gamma=0.5$.

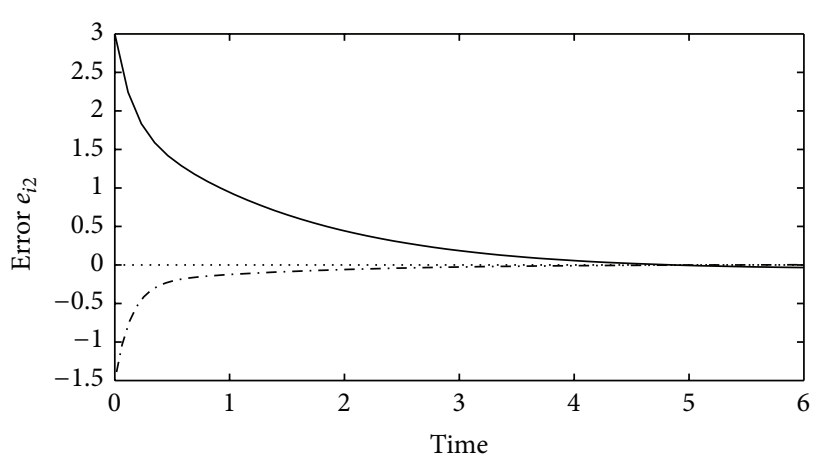

FIGURE 2: Synchronization error $e_{i 1}$ for the coupling delayed networks with $h=\gamma=0.5$.

state but also the delay-upper-bounded state to exploit all possible information when constructing the LyapunovKrasovskii functional. Our approach in Theorem 6 reduces the conservatism of the existing methods to a certain extent.

The aforementioned results can be extended to the case of bound constant time delay. If the time-varying delay $\tau(t)$ is a constant time delay in system (1), then fuzzy system (4) can be expressed in the following model:

$$
\begin{array}{r}
\dot{x}_{i}(t)=\sum_{l=1}^{r} \mu_{l}(\theta(t))\left[f\left(x_{i}(t)\right)+\varepsilon \sum_{j=1}^{N} c_{i j} \Gamma_{l} x_{j}(t)\right. \\
\left.+\varepsilon \sum_{j=1}^{N} \widetilde{c}_{i j} \widetilde{\Gamma}_{l} x_{j}(t-\tau)\right] .
\end{array}
$$

We obtain the following corollary.

Corollary 8. If there exist positive definite symmetric matrices $P_{k}, Q_{k}$, and $R_{k}(2 \leq k \leq N)$ and two arbitrary matrices $N_{1}$, 
TABLE 1: Upper bounds of $h$ for different $\varepsilon$ and $\gamma$.

\begin{tabular}{lcccc}
\hline Coupling strength $\varepsilon$ & 0.3 & 0.4 & 0.5 & 0.6 \\
\hline$\gamma=0$ & 1.832 & 1.136 & 0.813 & 0.637 \\
$\gamma=0.2$ & 1.461 & 1.053 & 0.716 & 0.562 \\
$\gamma=0.5$ & 1.263 & 0.851 & 0.641 & 0.514 \\
$\gamma=0.9$ & 0.953 & 0.710 & 0.560 & 0.473 \\
\hline
\end{tabular}

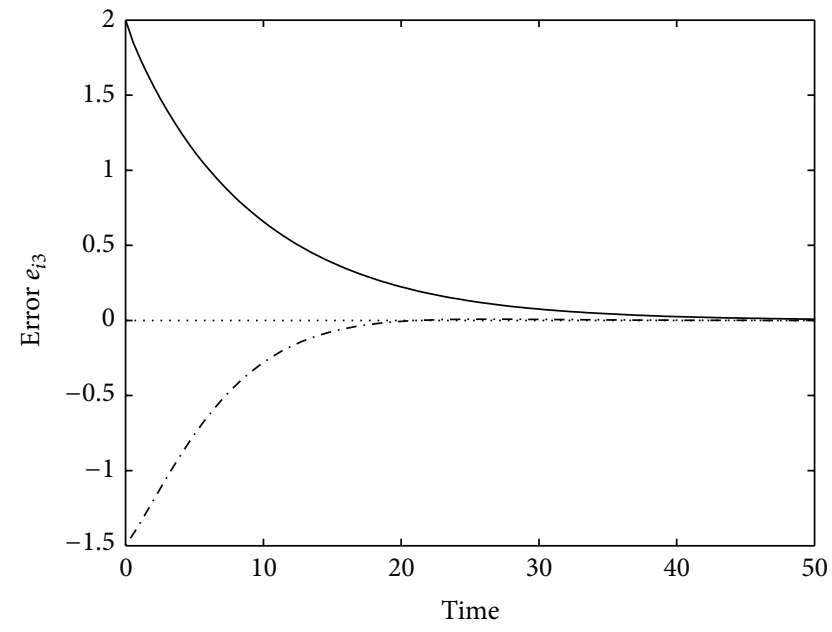

FIGURE 3: Synchronization error $e_{i 3}$ for the coupling delayed networks with $h=\gamma=0.5$.

$\mathrm{N}_{2}$ with appropriate dimensions, such that the following LMIs hold for all $l=1,2, \ldots, r$ :

$$
\Omega_{k}=\left[\begin{array}{cccc}
\Theta & \tilde{\lambda}_{k} \varepsilon P_{k} \widetilde{\Gamma}_{l}-N_{1}+N_{2}^{T} & \tau J^{T}(t) R_{k} & \tau N_{1} \\
* & -Q_{k}-N_{2}-N_{2}^{T} & \tau c \widetilde{\lambda}_{k} \widetilde{\Gamma}_{l}^{T} R_{k} & \tau N_{2} \\
* & * & -\tau R_{k} & 0 \\
* & * & * & -\tau R_{k}
\end{array}\right]<0
$$

where

$$
\Theta=P_{k}\left(J(t)+\varepsilon \lambda_{k} \Gamma_{l}\right)+\left(J(t)+\varepsilon \lambda_{k} \Gamma_{l}\right)^{T} P_{k}+Q_{k}+N_{1}^{T}+N_{1},
$$

then the asymptotic synchronization of system (22) in Definition 1 can be achieved.

Proof. Select a Lyapunov-Krasovskii function as

$$
\begin{aligned}
V_{k}(t)= & w_{k}^{T}(t) P_{k} w_{k}(t)+\int_{t-\tau}^{t} w_{k}^{T}(s) Q_{k} w_{k}(s) d s \\
& +\int_{t-\tau}^{t} \int_{\theta}^{t} \dot{w}_{k}^{T}(s) R_{k} \dot{w}_{k}(s) d s d \theta
\end{aligned}
$$

The rest of the proof is similar to that of Theorem 6; thus one can easily obtain the result. Thus it is omitted here. The proof is completed.

Remark 9. Corollary 8 presents new hybrid synchronization conditions for fuzzy complex networks with constant delays.
We consider the delay which involve general form of delays. Based on Lyapunov-Krasovskii function method, stability criteria are obtained in the form of LMIs which can be easily solved. And the results are usually less conservative than delay-independent ones especially when the size of the time delay is small.

\section{Numerical Example}

To illustrate the previous results we obtain, we consider the following complex dynamical networks with time-varying delays consisting of three nodes:

$$
\left[\begin{array}{c}
\dot{x}_{1}(t) \\
\dot{x}_{2}(t) \\
\dot{x}_{3}(t)
\end{array}\right]=\left[\begin{array}{c}
-x_{1} \\
-2 x_{2} \\
-3 x_{3}
\end{array}\right]
$$

which is asymptotically stable at $s(t)=0$, with Jacobin given by $J=\operatorname{diag}\{-1,-2,-3\}$. Assume that inner-coupling matrices are $\Gamma=\widetilde{\Gamma}=\operatorname{diag}\{1,1,1\}$, and the coupling configuration matrices are

$$
\begin{aligned}
& C=\widetilde{C}=\left[\begin{array}{lll}
-1 & 0.5 & 0.5 \\
0.5 & -1 & 0.5 \\
0.5 & 0.5 & -1
\end{array}\right], \\
& \mu_{1}(\theta(t))=\sin ^{2}\left(x_{1}(t)\right), \\
& \mu_{2}(\theta(t))=\cos ^{2}\left(x_{1}(t)\right) .
\end{aligned}
$$

The eigenvalues of $C$ and $\widetilde{C}$ are $\lambda(C)=\widetilde{\lambda}(\widetilde{C})=\{0,-1.5$, $-1.5\}$.

Using MATLAB LMI toolbox, the upper bounds on the time delay for different values of the coupling strength $\varepsilon$ can be obtained from Theorem 6 . A detailed comparison is given in Table 1, where the achieved upper bounds of time delay in the previous system are listed for their respective lower bounds.

Assume that the coupling strength is $\varepsilon=0.4$; it is found that the maximum delay bound is $h=0.73$ in [27], for which the synchronized states of the network are asymptotically stable. By Theorem 6 in this paper, however, it is found that the maximum delay bound for the synchronized states to be asymptotically stable is $h=0.851$. It can be seen that the method proposed in this paper is better.

For example, when the delay is $\gamma=0.5$, by employing the LMI Toolbox in Matlab, we simulate system (26) for given chosen initial parameter $\varepsilon=0.3$. We define the errors of synchronization as follows:

$$
e_{i j}(t) \triangleq x_{i j}(t)-x_{(i+1) j}(t), \quad i=1,2, j=1,2,3 .
$$


For random initial conditions, Figures 1, 2, and 3 show the synchronization errors between the states of node $i$ and node $i+1$ with $h=0.5$. We find that the synchronization errors converge to zero under the previous conditions.

\section{Conclusions}

In this paper, we have proposed a class of general complex dynamical network models based on T-S fuzzy theory and investigated hybrid synchronization of the proposed complex dynamical networks. Time-varying delays in network couplings of dynamical nodes have been considered. New delaydependent synchronization criteria in terms of LMIs have been derived based on an appropriate Lyapunov functional. Synchronization criteria are obtained in this paper which can be applicable to networks with different topologies and different sizes. Numerical simulation is also provided to illustrate the usefulness and advantage of the synchronization criteria.

\section{Acknowledgments}

This research was partially supported by the National Natural Science Foundation of PR China (Grant nos. 61104135, 61025015, 61174132, and 61134006), China Postdoctoral Science Fund (Grant no. 2012T50705), and Postdoctoral Fund of the Central South University. The authors also gratefully thank the Editor and the reviewers for their helpful comments and suggestions, which have improved the quality of this paper.

\section{References}

[1] A.-L. Barabási and R. Albert, "Emergence of scaling in random networks," Science, vol. 286, no. 5439, pp. 509-512, 1999.

[2] R. Albert and A.-L. Barabási, "Statistical mechanics of complex networks," Reviews of Modern Physics, vol. 74, no. 1, pp. 47-97, 2002.

[3] S. Boccaletti, V. Latora, Y. Moreno, M. Chavez, and D.-U. Hwang, "Complex networks: structure and dynamics," Physics Reports, vol. 424, no. 4-5, pp. 175-308, 2006.

[4] M. Newman, A.-L. Barabási, and D.J. Watts, Eds., The Structure and Dynamics of Networks, Princeton University Press, Princeton, NJ, USA, 2006.

[5] A. Arenas, A. Díaz-Guilera, and C. J. Pérez-Vicente, "Synchronization processes in complex networks," Physica D, vol. 224, no. 1-2, pp. 27-34, 2006.

[6] J. Lü, H. Leung, and G. Chen, "Complex dynamical networks: modeling, synchronization and control," Dynamics of Continuous, Discrete and Impulsive Systems B, vol. 11, pp. 70-77, 2004.

[7] J. Lü, X. Yu, and G. Chen, "Chaos synchronization of general complex dynamical networks," Physica A, vol. 334, no. 1-2, pp. 281-302, 2004.

[8] W. Lu and T. Chen, "Synchronization analysis of linearly coupled networks of discrete time systems," Physica D, vol. 198, no. 1-2, pp. 148-168, 2004.

[9] C. Li and G. Chen, "Synchronization in general complex dynamical networks with coupling delays," Physica A, vol. 343, no. 1-4, pp. 263-278, 2004.
[10] Z. Wang, Y. Wang, and Y. Liu, "Global synchronization for discrete-time stochastic complex networks with randomly occurred nonlinearities and mixed time delays," IEEE Transactions on Neural Networks, vol. 21, no. 1, pp. 11-25, 2010.

[11] J. Cao, Z. Wang, and Y. Sun, "Synchronization in an array of linearly stochastically coupled networks with time delays," Physica A, vol. 385, no. 2, pp. 718-728, 2007.

[12] $\mathrm{D} . \mathrm{Xu}$ and $\mathrm{Z}$. Su, "Synchronization criterions and pinning control of general complex networks with time delay," Applied Mathematics and Computation, vol. 215, no. 4, pp. 1593-1608, 2009.

[13] J. Lü and G. Chen, "A time-varying complex dynamical network model and its controlled synchronization criteria," IEEE Transactions on Automatic Control, vol. 50, no. 6, pp. 841-846, 2005.

[14] Z. Fei, H. Gao, and W. X. Zheng, "New synchronization stability of complex networks with an interval time-varying coupling delay," IEEE Transactions on Circuits and Systems II, vol. 56, no. 6, pp. 499-503, 2009.

[15] J. H. Koo, D. H. Ji, and S. C. Won, "Synchronization of singular complex dynamical networks with time-varying delays," Applied Mathematics and Computation, vol. 217, no. 8, pp. 39163923, 2010.

[16] T. Takagi and M. Sugeno, "Fuzzy identification of systems and its applications to modeling and control," IEEE Transactions on Systems, Man and Cybernetics, vol. 15, no. 1, pp. 116-132, 1985.

[17] J. Qiu, G. Feng, and J. Yang, "A new design of delay-dependent robust Ho filtering for discrete-time T-S fuzzy systems with time-varying delay," IEEE Transactions on Fuzzy Systems, vol. 17, no. 5, pp. 1044-1058, 2009.

[18] J. Qiu, G. Feng, and H. Gao, "Fuzzy-model-based piecewise $\mathscr{H} \infty$ static-output-feedback controller design for networked nonlinear systems," IEEE Transactions on Fuzzy Systems, vol. 18, no. 5, pp. 919-934, 2010.

[19] J. Qiu, G. Feng, and H. Gao, "Nonsynchronized-State estimation of multichannel networked nonlinear systems with multiple packet dropouts Via TS Fuzzy-Affine dynamic models," IEEE Transactions on Fuzzy Systems, vol. 19, no. 1, pp. 75-90, 2011.

[20] L. Wu and D. W. C. Ho, "Fuzzy filter design for Itô stochastic systems with application to sensor fault detection," IEEE Transactions on Fuzzy Systems, vol. 17, no. 1, pp. 233-242, 2009.

[21] L. Wu and W. X. Zheng, "L2-Lo control of nonlinear fuzzy itô stochastic delay systems via dynamic output feedback," IEEE Transactions on Systems, Man, and Cybernetics B, vol. 39, no. 5, pp. 1308-1315, 2009.

[22] L. Wu, X. Su, P. Shi, and J. Qiu, "Model approximation for discrete-time state-delay systems in the TS fuzzy framework," IEEE Transactions on Fuzzy Systems, vol. 19, no. 2, pp. 366-378, 2011.

[23] L. Wu and W. X. Zheng, "Weighted $H_{\infty}$ model reduction for linear switched systems with time-varying delay," Automatica, vol. 45, no. 1, pp. 186-193, 2009.

[24] L. Wu, X. Su, P. Shi, and J. Qiu, "A new approach to stability analysis and stabilization of discrete-time T-S fuzzy timevarying delay systems," IEEE Transactions on Systems, Man, and Cybernetics B, vol. 41, no. 1, pp. 273-286, 2011.

[25] Y. Tang, J. Fang, and M. Xia, "Synchronization of TakagiSugeno fuzzy stochastic complex networks with mixed delays," in Proceedings of the 2nd International Conference on Intelligent Computation Technology and Automation (ICICTA '09), vol. 2, pp. 810-813, 2009. 
[26] Y. Tang, J.-A. Fang, M. Xia, and X. Gu, "Synchronization of Takagi-Sugeno fuzzy stochastic discrete-time complex networks with mixed time-varying delays," Applied Mathematical Modelling, vol. 34, no. 4, pp. 843-855, 2010.

[27] H. Li, "Delay-distribution-dependent synchronization of T-S fuzzy stochastic complex networks with mixed time delays," in Proceedings of the Control and Decision Conference (CCDC), pp. 1629-1634, 2011.

[28] K. Gu, "An integral inequality in the stability problem of timedelay systems," in Proceedings of the 39th IEEE Confernce on Decision and Control, pp. 2805-2810, December 2000.

[29] S. M. Lee, O. M. Kwon, and J. H. Park, "A novel delay-dependent criterion for delayed neural networks of neutral type," Physics Letters A, vol. 374, no. 17-18, pp. 1843-1848, 2010.

[30] X. J. Wu and H. T. Lu, "Hybrid synchronization of the general delayed and non-delayed complex dynamical networks via pinning control," Neurocomputing, vol. 89, no. 15, pp. 168-177, 2012.

[31] S. Wang, Y. Yu, and M. Diao, "Hybrid projective synchronization of chaotic fractional order systems with different dimensions," Physica A, vol. 389, no. 21, pp. 4981-4988, 2010.

[32] Y. Dai, Y. Cai, and X. Xu, "Synchronization criteria for complex dynamical networks with neutral-type coupling delay," Physica A, vol. 387, no. 18, pp. 4673-4682, 2008. 


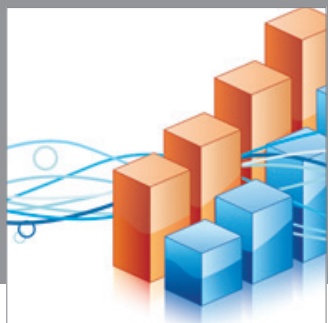

Advances in

Operations Research

mansans

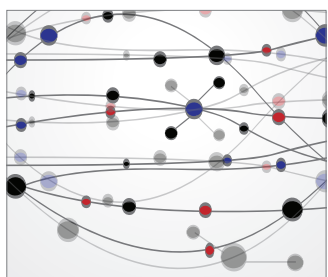

The Scientific World Journal
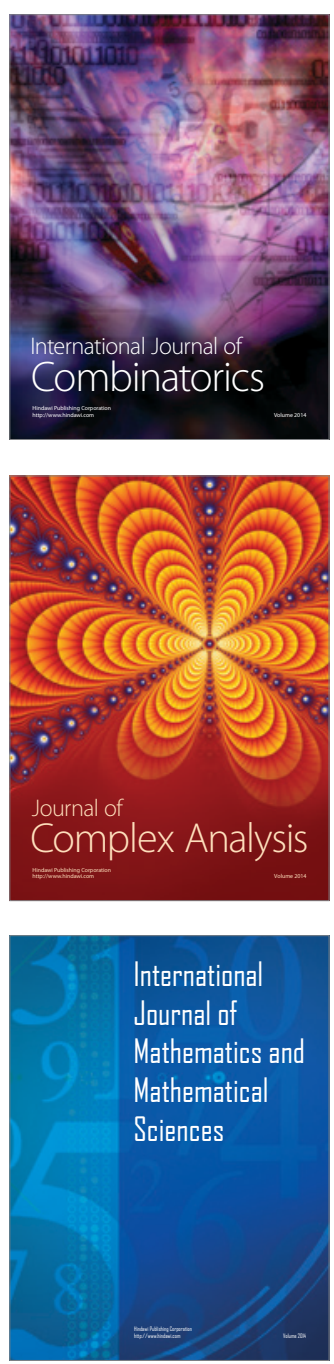
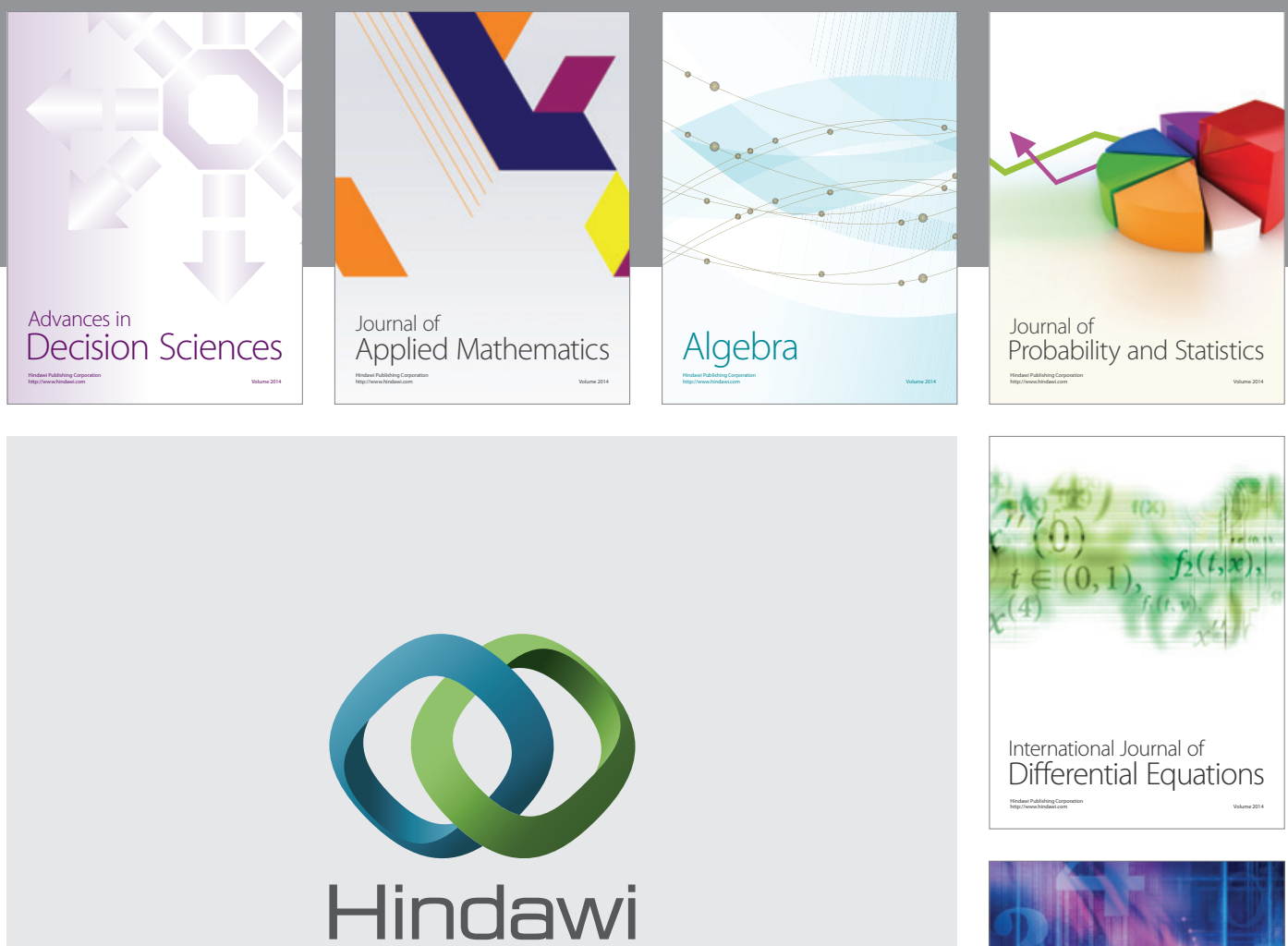

Submit your manuscripts at http://www.hindawi.com
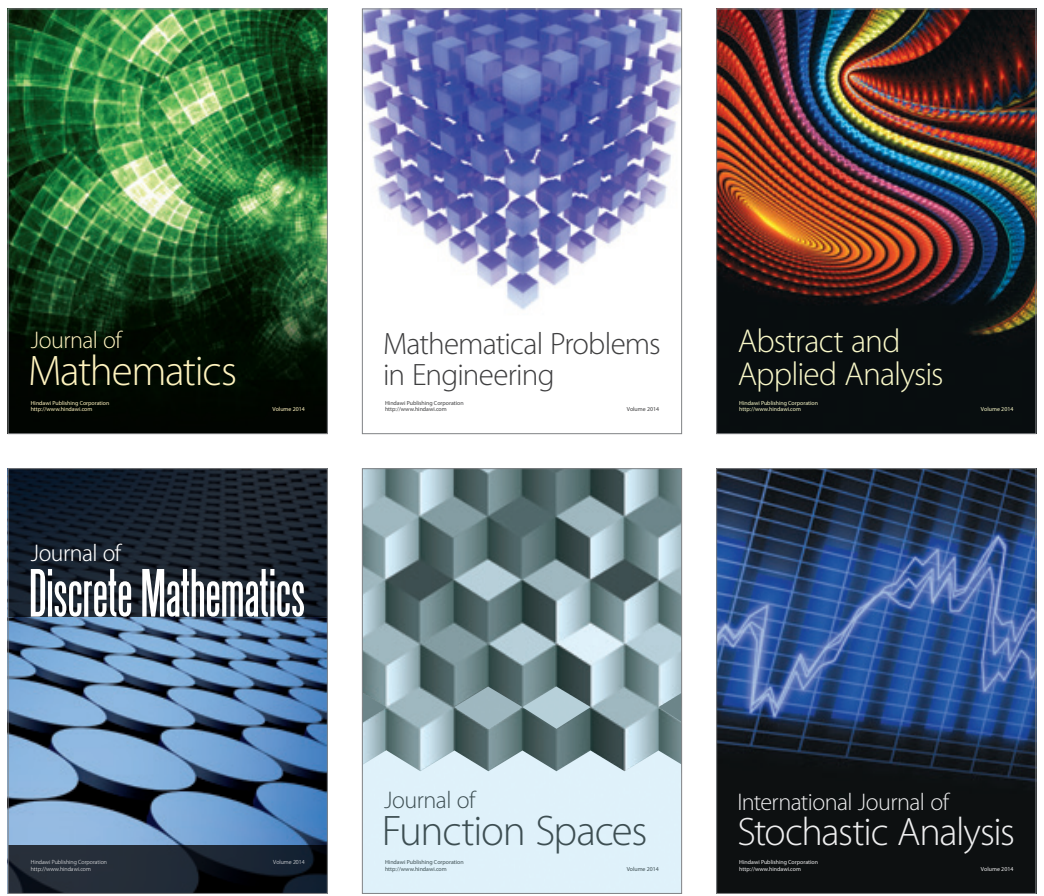

Journal of

Function Spaces

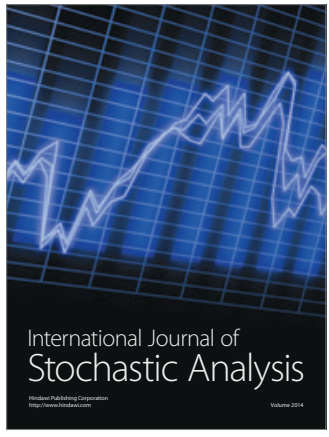

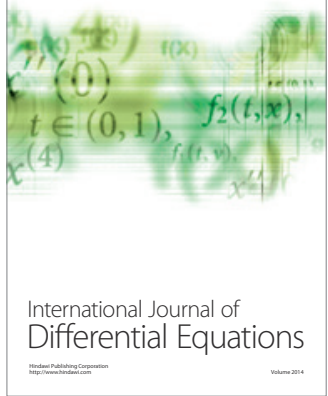
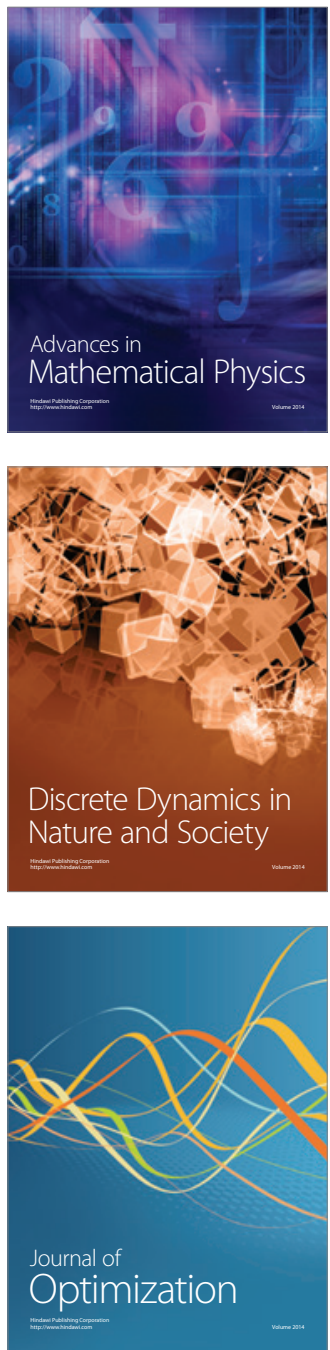